\title{
Dilational viscosity of Langmuir monolayers
}

\author{
Vladimir Kolevzon \\ Institute of Physical Chemistry, University of Karlsruhe, \\ 76128 Karlsruhe, Germany
}

March 3, 2018

\begin{abstract}
The dilational viscosity $\epsilon^{\prime}$ of the Langmuir monolayer is considered in a theoretical model taking into account an orientational effect of the dilational wave on the surface molecules. The surface tension treated in the framework of this model is supposed to be dependent on the degree of molecular ordering in the monolayer plane. This orientational order is described by the surface order parameter $\mathrm{Q}$ and the orientational part of the free energy which is given by Landau's expansion in powers of $\mathrm{Q}$. The magnitude of surface viscosity determined by the surface tension derivative $\partial \gamma / \partial Q$ is found to be in good accord with the experimentally observed $\epsilon^{\prime}$. The sign of $\epsilon^{\prime}$ is positive which indicates that increased ordering in the monolayer plane increases the surface tension.
\end{abstract}

PACS: 61.25.Mv; 68.10.-m; 68.10.Cr

Strong damping of capillary waves on the monolayer-water interface has been well known for many years. However the physical nature of the two surface viscosities which are included into a dispersion relation [1, 2] describing the propagation of surface waves still remains unclear. It has been suggested that the monolayer possesses up to three surface viscosities: dilational, shear in the interfacial plane and shear normal to the surface plane [1, 3]. Only two of them: dilational $\epsilon^{\prime}$ and transverse shear $\gamma^{\prime}$ do couple to surface modes which scatter light [2]. These surface viscosities were introduced as the response functions which appear after expansion of the complex moduli $\gamma, \epsilon$, to linear order in $\omega[1]$. Hence the dilational modulus is usually written as a superposition of the dilational surface viscosity $\epsilon^{\prime}$ and the surface elasticity 
$\epsilon_{0}[1]$, 3]:

$$
\epsilon=\epsilon_{0}+i \omega \epsilon^{\prime}
$$

The surface viscosity $\epsilon^{\prime}$ so introduced is a pure phenomenological property which was suggested as the surface excess quantity [3, 4].

Only for two-component systems of soluble surfactants is there a strong theoretical basis for the dilational surface viscosity [5, 6]. In such a system the surface viscoelasticity appears as a result of the competition between adsorption and diffusion in the concentrational boundary layer beneath the surface. The main result of this formalism is that $\epsilon^{\prime}$ is not an independent property but is a function of some relaxation time $\tau$ which depends on the diffusion coefficient $\mathrm{D}$ and the slope of the adsorption isotherm $\frac{d C}{d \Gamma_{s}}[5,6]$ :

$$
\epsilon^{\prime}=\frac{\epsilon_{0} \tau}{\omega\left(1+2 \tau+2 \tau^{2}\right)}
$$

where $\tau=\frac{d C}{d \Gamma_{s}} \sqrt{D / \omega}$, (C is the bulk concentration and $\Gamma_{s}$ the surface concentration). However for an insoluble (spread) monolayer on a liquid substrate there is no adequate theoretical description for the dilational surface viscosity. At the same time the experimental findings of negative $\epsilon^{\prime}$ in some liquid systems [0, 8] indicate that some important physical processes are not accounted for in the theoretical formalism of [1, 6].

Our previous papers concerned the dilational viscosity of various liquid surfaces [9, 10]. It was demonstrated that the surface dilational modulus can be described by relaxation of fluctuations of temperature and surface charge density. Unfortunately the dilational viscosity calculated from those models appears to be a few orders of magnitude smaller than that observed in experiments on different liquid systems [7, 8]. The present paper demonstrates that the orientational effect of the surface wave on long molecules can be the reason for the dilational viscosity of the liquid-monolayer interface.

The orientational effect of a shear flow on long molecules in the liquid bulk has been known for many years [11]. However such an orientation of molecules in the surface layer has not yet been reported. We concentrate upon a monolayer being in a liquid-expanded (LE) state where the surface area per molecule is relatively large according to the surface isotherm [12]. Due to this fact and strong electrostatic interaction between the molecular head groups and water the long axes of molecules are not oriented along the surface normal but comprise some angle to it. 
Unfortunately LE state is not too much studied experimentally compared to a condensed state. Therefore we will use the results of theoretical predictions on order parameters in LE state. One of them [13] develops a concept of orientational order in a monolayer comprising hard rods grafted on the surface. The main result is that molecular alignment increases continuously with increasing surface concentration. Since the molecular azimuths are not arbitrary but are defined by the tilt angle, the molecular alignment in the surface plane is induced by collective tilt. A numerical analysis of flexible molecules [14] shows that in-plane ordering in a liquid phase can be even enhanced by chain length. All these papers presume that surface fluctuations destroy the in-plane long range order. We will give a highlight to the effect of dilational waves on the surface order. As it will be shown below a very small magnitude of the order parameter is sufficient for our model.

As mentioned in [13], a monolayer formed by amphiphile diblock copolymer on water surface can be a realization of the model describing alignment of mobile rods. Another experimental situation relevant to the present context is monolayer of a nematogen on water studied in [15]. At low surface concentrations nematic molecules lie on water but the molecules interact with each other as in a 3-D nematic.

In the present context we suggest that projections of molecules display a quasi long-range ordering in the surface plane. In the case of a high tilt angle the short axis of a molecule nearly coincide with its surface projection. Let us consider the surface footprint of a tilted molecule (see Fig. 1). The long axis of the molecular "cross-section" of a length L makes an angle $\phi$ with wavevector $\mathrm{q}$ (supposed to be along the x-axis) of the dilational wave. This molecule experiences an orientation torque induced by the longitudinal gradient $\mathrm{du}_{x} / \mathrm{dx}$ of velocity in the dilational wave as it was suggested in [16]. If the center of the molecule moves with the liquid then the two ends will move with different relative velocities $u_{1}$ and $u_{2}$ :

$$
u_{1}-u_{2}=\delta u=\frac{d u_{x}}{d x} L \cos \phi
$$

The transverse (to the molecule axis) component of the relative velocity gives rise to rotation of the molecule with an angular velocity $\Omega=\dot{\phi}$

$$
\Omega=-\frac{d u_{x}}{d x} \sin 2 \phi
$$


where negative sign means that the molecule rotates towards to decrease in $\phi$.

The angular velocity of the molecular rotation is proportional to the gradient of surface velocity whose magnitude is small and oscillating: $u \sim$ $u^{\prime} \exp (i(\omega t-q x))$. The effect is superposed with a strong anisotropy of nematic in-plane ordering supposed in the present text. The order parameter tensor can be introduced in the pure $2-\mathrm{D}$ case, analogously to 3 -D case [17, as : $Q_{i j}=Q\left(n_{i} n_{j}-\frac{1}{2} \delta_{i j}\right)$ where $\mathrm{i}=1,2, \mathrm{n}_{i}$ is the nematic director lying in the surface plane. Due to the surface wave the order parameter tensor comprises two parts: steady $\mathrm{Q}_{i j}^{0}$ and an oscillating one $\mathrm{Q}_{i j}^{\prime}$.

In order to find out the equation describing the behavior of $\mathrm{Q}^{\prime}$ we use the well-known phenomenological equation [17]:

$$
\frac{d Q_{i j}}{d t}=\lambda s_{i k}-\mu \frac{\partial F}{\partial Q_{i j}}
$$

where $\mathrm{F}$ is the free energy density, $\lambda$ is a proportionality coefficient, $\mathrm{s}_{i k}$ is the tensor of viscous stresses and $\mu$ is some coefficient having the dimension of viscosity. Usually the free energy is expanded in powers of $Q$ and the expansion contains the terms proportional to the second, third and fourth power of $\mathrm{Q}$ (see for example [17). The main feature of the 2-D case is an absence of a cubic term in the energy expansion due to the symmetry in the surface plane. Thus the free energy density is written as:

$$
F=A Q_{i j} Q_{i j}+B Q_{i j} Q_{i j} Q_{k l} Q_{k l}
$$

where $\mathrm{A}$ is the temperature dependent coefficient changing sign at the point of an isotropic-nematic transition and $\mathrm{B}>0$, and the summation is taken over repeated indexes. In principle, it might be possible to calculate $\mathrm{A}$ and $\mathrm{B}$ in the $2-\mathrm{D}$ case as it is done in the bulk nematic [20]. However, an anisotropic interaction potential is essentially unknown to the present study. Therefore the coefficients A and B will be kept without explicit expressions.

Since we have only one non-zero component of the stress tensor $: s_{11}=$ $\frac{2}{3} \frac{\partial u_{x}}{\partial x}$ (see [11]) $\mathrm{Eq}(6)$ is simplified

$$
\frac{d Q_{11}}{d t}=\lambda \frac{2}{3} \frac{\partial u_{x}}{\partial x}-\frac{1}{\tau} \frac{\partial f}{\partial Q_{11}}
$$

where $\mathrm{f}$ is dimensionless energy density $f=F / F_{0} / V_{0}$ and $\tau=\mu F_{0} / V_{0}$ is some relaxation time. The derivative $\partial F / \partial Q_{i j}$ can be found from the 
substitution of the averaged plus the disturbed component of the order parameter tensor into $\mathrm{Eq}(6)$. However we simplify the situation suggesting that the molecular axes are oriented in the surface plane either parallel to the wavevector $\mathbf{q}$ of the dilational wave or perpendicular to it. Then the disturbed component of the order parameter tensor has a diagonal form, i.e. only two non-zero components: $\mathrm{Q}_{11}^{\prime}$ and $\mathrm{Q}_{22}^{\prime}$ whereas $\mathrm{Q}_{22}^{\prime}=-\mathrm{Q}_{11}^{\prime}=Q^{\prime}$. Thus the free energy derivative is

$$
\frac{\partial f}{\partial Q_{11}}=\frac{\partial}{\partial Q^{\prime}}\left(A\left(Q_{0}+Q^{\prime}\right)^{2}+B\left(Q_{0}+Q^{\prime}\right)^{4}\right)
$$

where $\mathrm{Q}_{0}$ is the steady part of the order parameter and $\mathrm{Q}^{\prime}$ is the magnitude of an oscillating part. Then $\mathrm{Eq}(\bar{\nabla})$ can be re-written to the first order in $\mathrm{Q}^{\prime}$

$$
\frac{d Q}{d t}=i \omega Q^{\prime}=-\frac{1}{\tau} Q^{\prime}\left(2 A+12 B Q_{0}^{2}\right)+\lambda \frac{2}{3} \frac{\partial u_{x}}{\partial x} .
$$

$\lambda$ is a phenomenological coefficient such that the term $\lambda \frac{\partial u_{x}}{\partial x}=\langle\Omega\rangle$ expresses the mean torque exerted on the order parameter by the dilational wave. Following Ref [19] this is plausible to suppose the coefficient $\lambda$ is the magnitude of the mean angular velocity averaged over all molecular orientations: The averaging gives $\lambda=1$ if the angular velocity in the form of $\mathrm{Eq}(\mathbb{4})$ is used. Due to its phenomenological origin, $\lambda$ is not inevitably equal to 1 but may depend of molecular shape, or diffusion coefficient, therefore only an approximation $\lambda \approx 1$ will be used. Hence $Q^{\prime}$ is

$$
Q^{\prime}=\frac{2}{3} \frac{\partial u_{x}}{\partial x} \frac{\tau}{i \omega \tau+2 A+6 B Q_{0}^{2}}
$$

The amplitude of $\mathrm{Q}^{\prime}$ is $\omega$ dependent if the typical wave frequency and relaxation time are such that: $\omega \sim \tau$. We remind that only the real part of the complex $\mathrm{Q}^{\prime}$ is physically meaningful; a phase of $\mathrm{Q}^{\prime}$ corresponds to some angle between the wavevector $\mathbf{q}$ and the averaged orientation of the director. The effect of this phase shift is an additional wave damping on the surface possessing some anisotropy. Our situation strongly resembles the propagation of longitudinal acoustic waves through an anisotropic liquid which was considered by Frenkel in [11]. He demonstrated that the components of the order tensor are proportional to the longitudinal gradient of velocity in a sound wave. If the wave frequency is comparable to the characteristic relaxation time then the order tensor and the velocity gradient are connected via the usual Maxwell formula describing the viscoelastic relaxation of molecules subjected to oscillating disturbances. Such viscoelasticity 
points to the existence of a phase shift between a disturbance and the media response [11].

Our goal is to find the surface elastic modulus which can be written as [6]:

$$
\epsilon=-\rho_{s} \frac{\partial \gamma}{\partial \rho_{s}}=-\rho_{s} \frac{\partial \gamma}{\partial Q} \frac{\partial Q}{\partial \rho_{s}}
$$

where $\gamma$ is the surface tension and $\rho_{s}$ is the surface concentration. The linkage between disturbances of the surface concentration and the surface velocity is found from the conservation of matter at the surface [5, 6]:

$$
\frac{\partial \rho_{s}}{\partial t}=D_{s} \frac{\partial^{2} \rho_{s}}{\partial x^{2}}-\rho_{s 0} \frac{\partial u_{x}}{\partial x}
$$

where $\rho_{s 0}$ is an equilibrium surface concentration. We are looking for the surface concentration in the form of a wave: $\rho_{s}^{\prime} \sim e^{i(\omega t-q x)}$. Substitution in $\mathrm{Eq}(12)$ yields:

$$
\rho_{s 0} \frac{\partial u_{x}}{\partial x}=\rho_{s}^{\prime}\left(-i \omega+D_{s} q^{2}\right) \approx-i \omega \rho^{\prime}
$$

where we supposed the surface diffusion coefficient $\mathrm{D}_{s}$ to be nearly equal to that of the bulk $\left(\mathrm{D} \approx 10^{-5} \mathrm{~cm}^{2} / \mathrm{s}\right)$ and typical wave frequencies are such that $\omega>>D_{s} q^{2}$ [2, 5]. It is easy to see that the second derivative in Eq(11) is:

$$
\frac{\partial Q^{\prime}}{\partial \rho_{s}}=-\frac{2}{3} \frac{i \omega \tau}{\rho_{s 0}\left(i \omega \tau+2 A+12 B Q_{0}^{2}\right)}
$$

where a negative sign means that $\mathrm{Q}$ is a decreasing function of the surface concentration. This is a typical signature of surface fluctuations; they tend to destroy a long range nematic order whose order parameter usually grows with $\rho_{s}$ in the absence of fluctuations 13.

Some papers (21] and references herein) postulate the existence of a nematic ordering induced on the surface of a liquid crystal being in an isotropic state having $\mathrm{Q} \equiv 0$. This surface ordering treated in [21] is due to the contact with a solid wall inducing a molecular alignment rapidly decaying into the liquid bulk. In 21] the free surface energy has been written using the Landau expansion over the in-plane order parameter. Note that liquid crystals have relatively large penetration depth of the surface ordering while the ordering in spread monolayers is strictly confined to the first surface layer. Therefore the difference from liquid crystals is an absence of the Frank elastic modulus describing the reaction of the bulk on the surface ordering. 
We establish now an analytical dependence of the surface tension on the surface order parameter. For the Langmuir monolayer only the surface has some ordering-the liquid bulk is essentially isotropic. Therefore the orientational part of the surface free energy is given by the integration of F over the liquid depth: $F_{s}=\int_{0}^{\infty} F(Q) \delta(z) d z=\xi F\left(Q_{s}\right)$, where $\delta(z)$ is the delta function and $\mathrm{Q}_{s}$ is the magnitude of the order parameter on the surface and $\xi$ is some correlation length. Thus the orientational part of surface free energy density is written as:

$$
F_{s}=\xi k_{B} T N_{A} \rho_{b} / M_{m}\left(A Q_{s}^{2}+B Q_{s}^{4}\right)=k_{B} T N_{A} \frac{\rho_{s}}{M_{m}} F(Q),
$$

where $\mathrm{M}_{m}$ is the molecular weight and the relation $\rho_{s}=\xi \rho_{b}$ connects the number of particles in the surface and the bulk. It should be borne in mind that only a minimum of the function $\mathrm{F}(\mathrm{Q})$ corresponds to physically stable state [17, 22] and minimization with respect to $\mathrm{Q}$ gives: $F_{\min }=\mathrm{A}$ $\mathrm{Q}_{0}^{2} / 2$, where $\mathrm{Q}_{0}^{2}=-\mathrm{A} / 2 \mathrm{~B}[22]$. It is apparent that in the $2-\mathrm{D}$ case we have a continuous phase transition instead of the first order transition in the bulk liquid crystal.

The derivative $\partial \gamma_{0} / \partial Q$ can be found from a general thermodynamic equation relating the surface tension and the surface excess density of mass $\Gamma_{s}$ [12]:

$$
d \gamma=-\Gamma_{s} d \mu_{s}
$$

where $\mu_{s}$ is the surface chemical potential. Note that the entropy term $\mathrm{S}_{s} \mathrm{dT}$ is omitted due to isothermal character of our effects. The chemical potential is $\mu_{s}=\left(\partial F_{s} / \partial N\right)_{A}$, by the definition [22] and $\Gamma_{s} \equiv \rho_{s}$ for any insoluble film. The derivative $\partial \gamma / \partial Q$ is

$$
\frac{\partial \gamma}{\partial Q}=-\rho_{s} \frac{\partial \mu_{s}}{\partial Q}=-\rho_{s} k_{B} T \frac{\partial F(Q)}{\partial Q}
$$

The variations in the surface mass density upon a phase transition are negligible.

As we mentioned above only a minimum in the free energy is realized in the equilibrium. One might think that $\partial F / \partial Q$ is zero as it should be in the minimum of a function. However this is not so due to deviations from the equilibrium induced by surface wave. To calculate $\partial F / \partial Q$ one should take into account the changes in surface area (and surface pressure) due to dilational wave. This means that the position of a minimum in the free 
energy and the corresponding order parameter $\mathrm{Q}_{0}$ also would change upon surface compression (rarefaction) due to a strong pressure dependence of A. Therefore we can take the $\mathrm{Q}_{0}$ derivative, having in mind that the position of minimum shifts with the change in $\mathrm{A}: \partial F / \partial Q_{0}=-A Q_{0}$. This derivative is negative $\left(\mathrm{Q}_{0}>0, \mathrm{~A}<0\right.$ in nematic phase $)$ and the minimum of the free energy is lowered upon an increase in $\mathrm{Q}_{0}$. Hence the $\mathrm{Q}$ derivative of the tension is positive:

$$
\partial \gamma / \partial Q=\rho_{s} / M_{m} N_{A} k_{B} T|A| Q_{0}
$$

It turns out that the derivative $\partial \gamma / \partial Q$ is positive for the whole Qrange and the surface viscosity is also positive according to $\mathrm{Eq}(11)$ where $\partial Q / \partial \rho_{s}<0$. Searching additional physical arguments in favor of an increasing $\gamma(\mathrm{Q})$ dependence one can return to $\mathrm{Eq}(11)$ introducing surface elasticity in the framework of orientational model. We may perturb a film by a small horizontal displacement which rarefies locally the monolayer and decreases the surface concentration. At the same time the surface expansion would increase the surface order parameter due to the orientational effect of the dilational wave discussed above. Negative sign in the definition of $\epsilon$ reflects the feed-back reaction of a perturbed surface element. For instance, one may expect the surface tension to decrease on expansion of the surface which is accompanied by some increase in Q. In this case a restoring force would act outward i.e. from the surface region with lower $\gamma$ to those regions having higher tension. Thus a decreasing $\gamma(\mathrm{Q})$ dependence would lead to a semi-infinitive expansion and the film instability. On the contrast, having supposed an increasing $\gamma(\mathrm{Q})$ dependence the surrounding film would tend to compress the surface element which was expanded initially. This ensures that increasing $\gamma(\mathrm{Q})$ provides the monolayer stability setting up $\partial \gamma / \partial Q>0$ and positive $\epsilon^{\prime}$.

The modulus of dilational viscosity given by $\epsilon^{\prime}=\Im[\epsilon] / \omega$, found from Eqs. (11), (14), (18)

$$
\epsilon^{\prime}(\omega)=\frac{k_{B} T N_{A} \rho_{s}|A| Q_{0}}{|\omega| M_{m}} \Im\left[\frac{\imath \tau}{\imath \tau+2 / \omega\left(A+6 B Q_{0}^{2}\right)}\right]
$$

is plotted in Fig 2 for different time constants $\tau$ using a typical value of the surface concentration $\rho_{s} \approx 1 \mathrm{mg} / \mathrm{m}^{2}$. This plot ensures that the dilational viscosity exhibits a strong $\omega$ dependence for some $\tau>10^{-5} \mathrm{~s}$ while for shorter time constants $\epsilon^{\prime}$ is nearly independent of $\omega$. 
Surface viscoelastic properties of glycerol mono-oleate monolayers on water were studied by surface light scattering in [23]. Surface properties were deduced from a many parametrical fit to the measured autocorrelation functions. Although the surface viscosity was introduced in the framework of a phenomenological model (see above) $\epsilon^{\prime} \approx 10^{-} 4 \mathrm{mN} \mathrm{s} / \mathrm{m}$ was found. The dilational viscosity inferred from the fit was sensitive to the monolayer compression: the increase in $\epsilon^{\prime}$ about an order of magnitude upon the monolayer compression have been reported.

Our analysis shows that the dilational surface viscosity appears in the form of oscillations (with some relaxation time) of the order parameter which take place due to the passage of the dilational wave. This surface anisotropy provides a phase shift between the velocity gradient and the in-plane director. The surface dilational viscosity introduced in our model is positive and drops to zero at the point where $\mathrm{A} \geq 0$ and $\mathrm{Q}=0$ i.e a point of the surface phase transition of a second order. The main assumption of the present paper is the existence of nematic ordering in the liquid-expanded state. However the present model of surface viscosity can be modified for hexatic ordering in Langmuir monolayers.

Fruitful discussions were shared with E.I. Kats.

\section{References}

[1] L.Kramer, J. Chem. Phys. 55, 52097 (1971)

[2] D.Langevin (Ed.), Light scattering by liquid surfaces and complementary techniques (Dekker, New York, 1992)

[3] F.C. Goodrich, Proc. R. Soc. London A 374, 341 (1981)

[4] M.Baus and C.F.Tejero, J. Chem. Phys. 78, 1, 483 (1983)

[5] V.G. Levich, Physicochemical Hydrodynamics Prentice Hall, N. J. (1962)

[6] E.H.Lucassen-Reynders and J.Lucassen, Adv. Colloid. Interface Sci. 2, 347 (1969)

[7] J.C.Earnshaw and E.McCoo, Langmuir 11, 1087 (1995) 
[8] V.Kolevzon and G.Gerbeth, J.Phys. D:Appl. Phys. 29, 2071, (1996)

[9] V.Kolevzon, Phys. Lett A, 230, 358 (1997)

[10] V.Kolevzon, unpublished (1997)

[11] J. Frenkel, Kinetic Theory of Liquids Dover, New York (1955)

[12] Arthur W. Adamsom, Physical Chemistry of Surfaces John Wiley \& Sons (1967)

[13] A. Halperin, S. Alexander and I. Schechter, J. Chem. Phys. 86, 6550, (1987)

[14] F. Schmid and M. Schick, J. Chem. Phys. 102, 2080, (1995)

[15] K. A. Suresh, A. Blumstein and F. Rondelez, J.Phys. (Paris) 46, 453 (1985)

[16] J. Priede, private communication (1997)

[17] P. G. de Gennes, The Physics of Liquid Crystals Clarendon Press, (Oxford) (1974)

[18] W. Maier and A. Saupe, Z. Naturforschg., 15a, 287, (1960)

[19] M. J. Stephen and J. P. Straley, Rev. Mod. Phys., 46, 4, 670 (1974)

[20] J. Katriel, G.F. Kventsel, G.R. Luckhurst, and T.J. Sluckin, Liquid Crystals 1, 337 (1986).

[21] T.J. Sluckin and A. Poniewierski, in Fluid Interfacial Phenomena ed. C.A. Croxton (Wliey, N.Y., 1985)

[22] L.D. Landau and E.M. Lifshitz, Statistical Physics Part 1 (Pergamon, Oxford, 1980)

[23] J C Earnshaw, R C McGivern and P J Winch, J. Phys. France 49, 1271 (1988) 


\section{Captions}

Fig.1 Orientational effect of the velocity field in the surface wave on the cross section of a molecule in Langmuir monolayer. $q$ is the wavevector of the dilational wave. The dashed lines show the lines of equal phase of velocity in longitudinal wave. Surface velocity remains constant along the lines but varies perpendicularly to them giving rise to the gradient $\mathrm{du}_{x} / \mathrm{dx}$ on the surface.

Fig.2 Surface dilational viscosity $\epsilon^{\prime}(\omega)$ calculated just near the surface phase transition for different relaxations times $\tau$. $\tau$ are labeled near each curve in s. The following values of the parameters are used: $\mathrm{Q}=0.1$, $|A|=0.4, \mathrm{~B}=1$ 

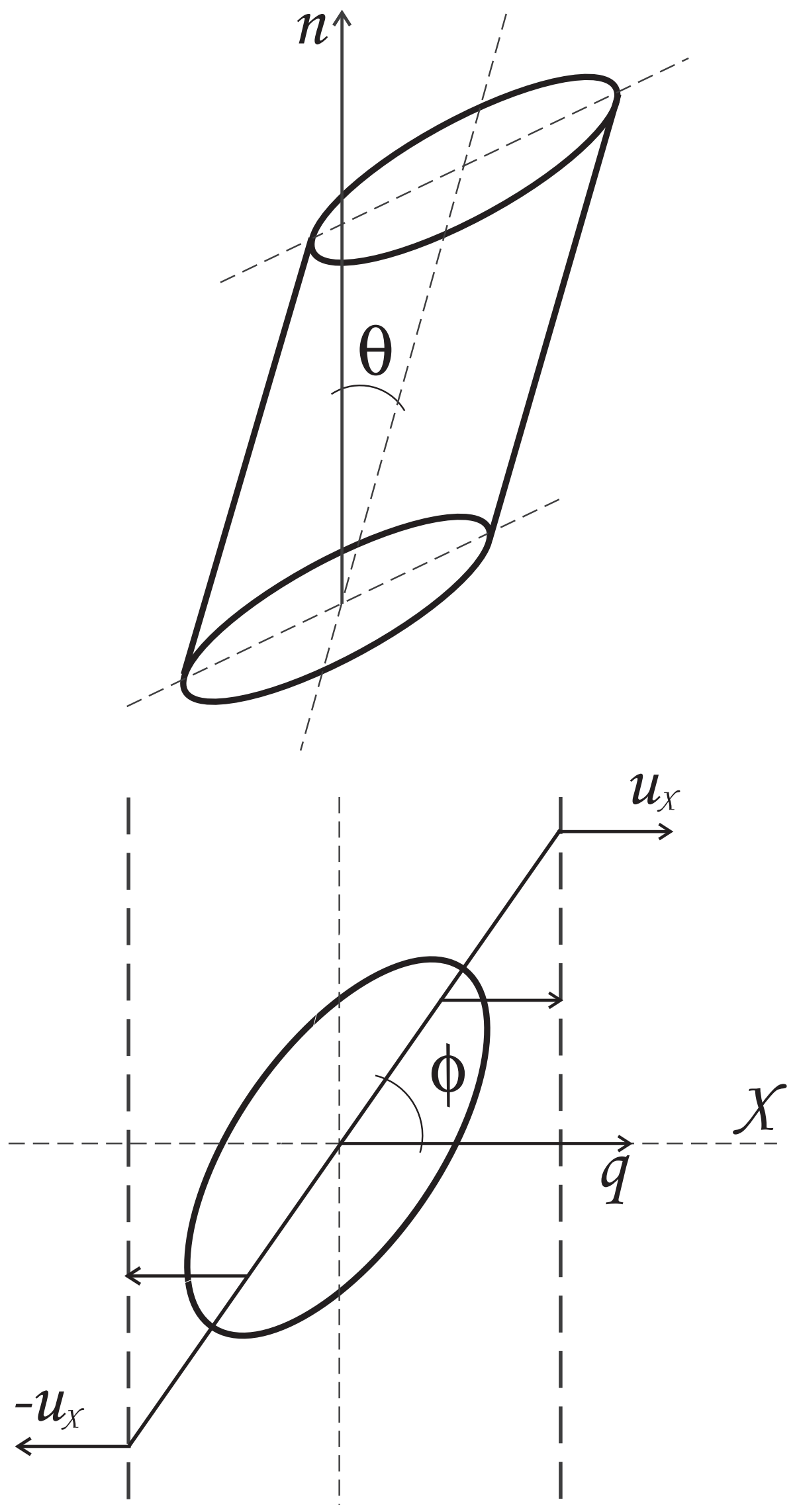


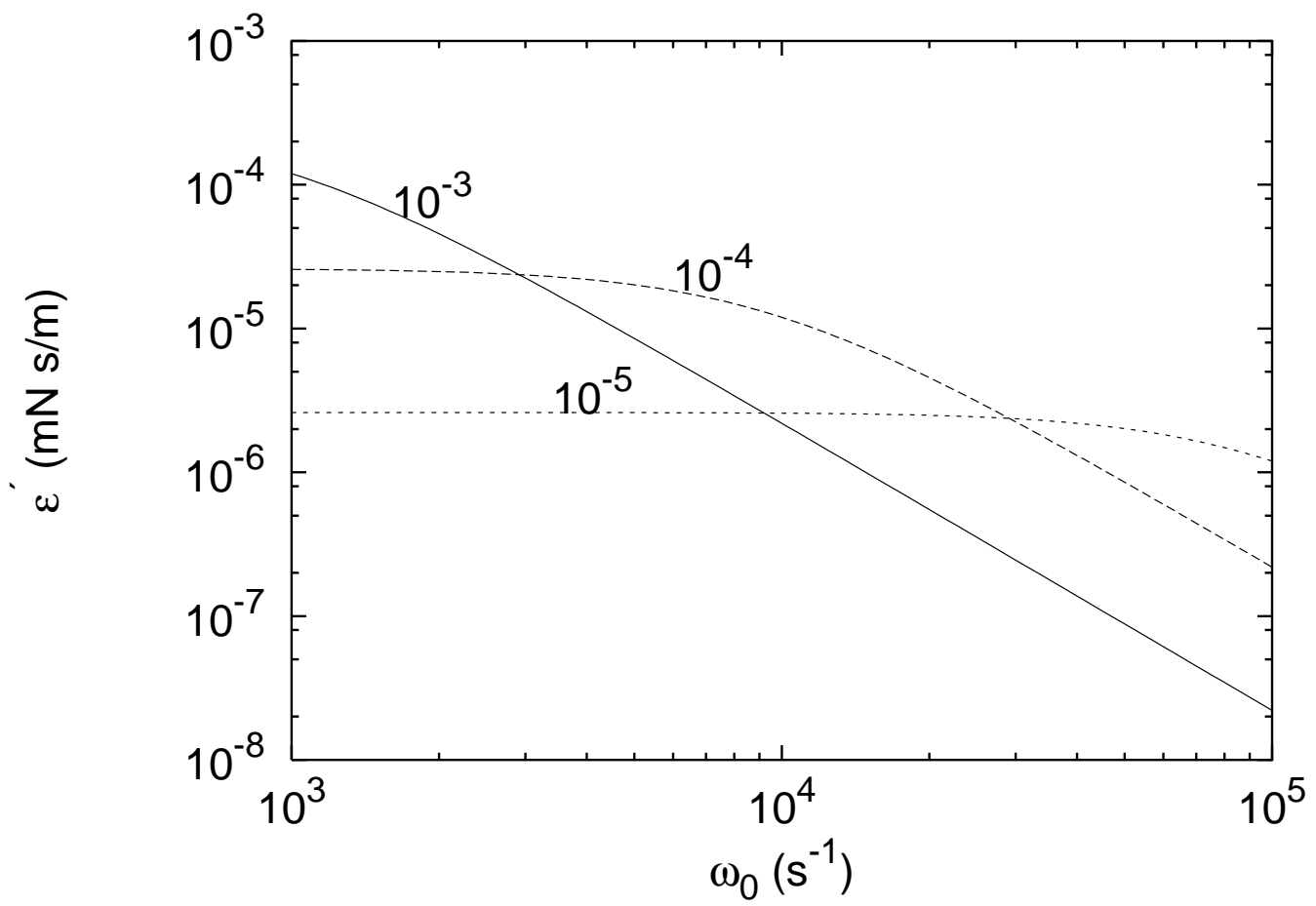

